\title{
Comparison of asthma control before and during the SARS-CoV-2 pandemic in children 3-18 years of age presenting to the pediatric pulmonary clinic at Brookdale Hospital Medical Center
}

\author{
Shah Mahrukh ${ }^{1}$, Mohammed Alsabri ${ }^{1}$, Farouk Al-Qadasi2 ${ }^{2}$, Saadia Malik ${ }^{3}$, Khalid \\ Ahmad $^{4}$, Carolyn springer ${ }^{1}$, Kusum Viswanathan ${ }^{1}$, and Fernanda Kupferman ${ }^{1}$ \\ ${ }^{1}$ Brookdale University Hospital and Medical Center \\ ${ }^{2}$ International Organization of Migration \\ ${ }^{3}$ Saba University School of Medicine \\ ${ }^{4}$ Brookdale University Hospital and Medical Center Department of Pediatrics
}

February 17, 2022

\begin{abstract}
OBJECTIVE: To determine asthma control during the SARS-CoV-2 pandemic in a minority pediatric population at a community hospital. BACKGROUND: During the pandemic, exposure to allergens and infectious agents has decreased during the pandemic due to heightened hygiene measures and primarily virtual visits. We examined the effect of the pandemic on pediatric asthma control. DESIGN/METHODS: Our study included 104 asthmatic children, 3-18 years of age. The mean age of patients was $9.7 \pm 3.8$. Subjects were assessed during the time period of March-August 2019 and 2020. Outcome variables included: rescue albuterol and systemic steroid use, physician visits (PMDv), emergency department visits (EDv), hospitalizations (H) for asthma exacerbation, pulmonology clinic visits, change in and adherence to controller therapy, spacer technique and BMI. RESULTS: During the pandemic, the majority of pulmonology clinic visits were via telemedicine. There was a significant difference in appropriate spacer technique and change in controller regimen, with improved technique and decreased requirement for step up controller therapy, during the pandemic There was no significant difference in BMI, adherence to controller therapy or the number of pulmonology visits. Additionally, there was an improvement in asthma control during the pandemic with less use of rescue albuterol, systemic steroids, H, EDv or PMDv for acute exacerbation. CONCLUSIONS: Overall, we found that asthma control has improved during the SARS-CoV-2 pandemic in terms of: reduced albuterol and systemic steroid use and decreased asthma exacerbations. The recent addition of telemedicine to patient care has not negatively affected asthma control in children.
\end{abstract}

\section{Hosted file}

DOC_Asthma_- No tables:figures .doc available at https://authorea.com/users/461058/articles/ 556846-comparison-of-asthma-control-before-and-during-the-sars-cov-2-pandemic-inchildren-3-18-years-of-age-presenting-to-the-pediatric-pulmonary-clinic-at-brookdalehospital-medical-center

\section{Hosted file}

DOC_Asthma_tables.doc available at https://authorea.com/users/461058/articles/556846comparison-of-asthma-control-before-and-during-the-sars-cov-2-pandemic-in-children3-18-years-of-age-presenting-to-the-pediatric-pulmonary-clinic-at-brookdale-hospitalmedical-center 
Figure 1a: Comparison of change and in controller therapy and appropriate spacer technique pre and During CoV-Sars-2 pandemic

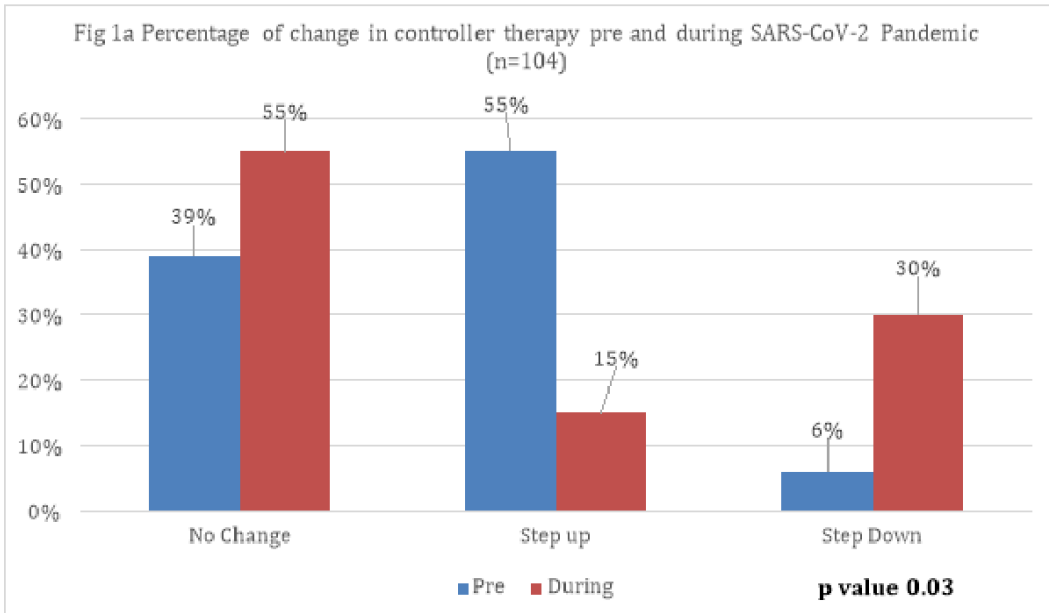


Figure 1b: Comparison of adherence to controller therapy and appropriate spacer technique pre and During CoV-Sars-2 pandemic

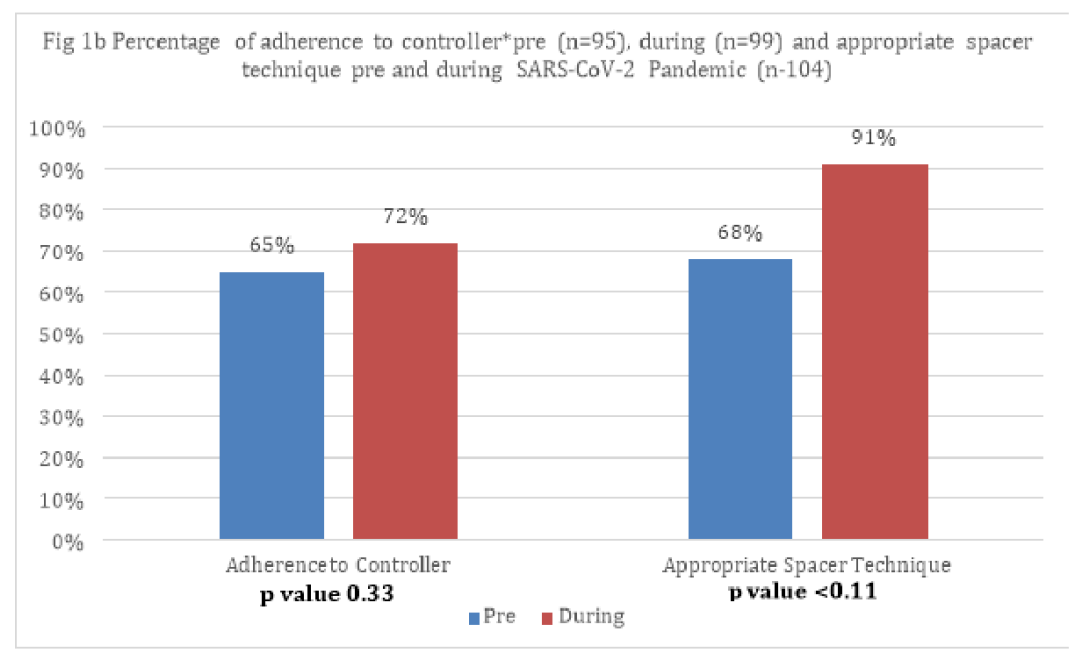

Figure 2: Comparison of asthma control variables pre and During CoV-Sars-2 pandemic 
Figure 2: Comparison of asthma control variables pre and During CoV-Sars-2 pandemic

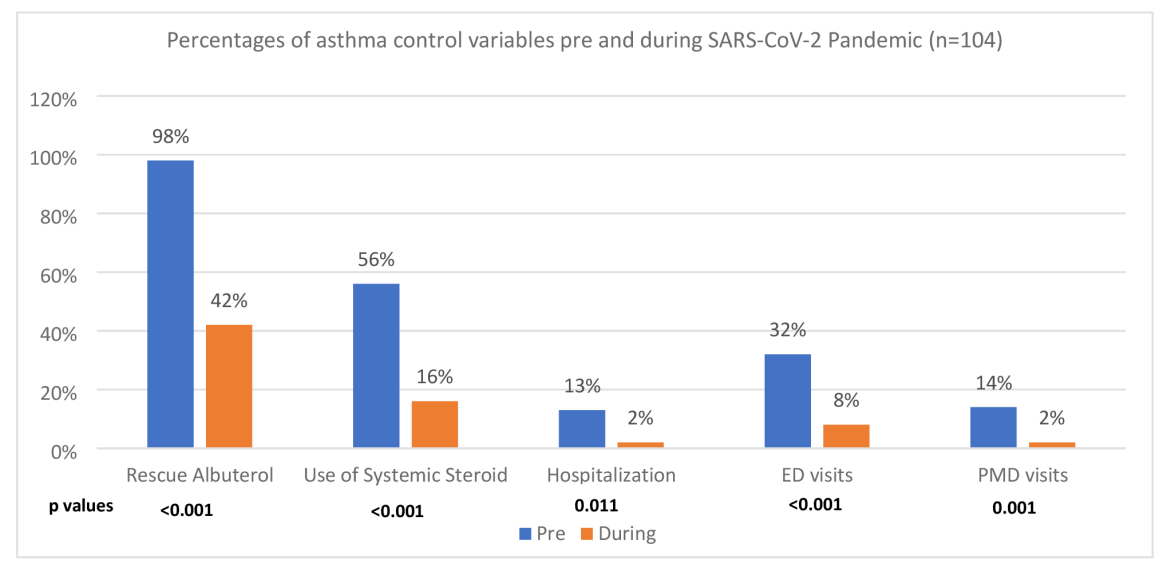

